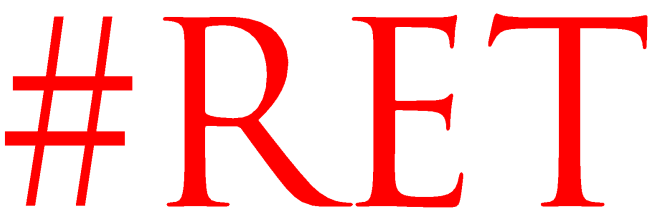

Revista Española de la Transparencia

Núm. 13. Segundo Semestre. Julio-diciembre de 2021, pp. 225-246

ISSN 2444-2607. Www.revistatransparencia.com

DOI: https://doi.org/10.51915/ret.114

\title{
El nivel educativo y los recursos de revisión como determinantes de la transparencia en las entidades federativas de México
}

\author{
César Omar Mora Pérez1 \\ Universidad de Guadalajara \\ Centro Universitario de Ciencias Económico-Administrativas \\ México \\ ORCID: $\underline{0000-0002-3917-1144}$
}

RECIBIDO: 30 de mayo de 2020 ACEPTADO: 28 de agosto de 2021

\begin{abstract}
RESUMEN: Este artículo plantea responder a la pregunta, si las inconformidades (recursos de revisión) y el nivel educativo en las entidades federativas de México tienen relación con la transparencia. Los diferentes apartados desarrollan los elementos conceptuales y teóricos que dan soporte a este planteamiento. Durante el desarrollo del documento se establece que las inconformidades tienen un efecto negativo en la transparencia, de igual manera, se plantea que el nivel educativo en educación media tiene un efecto positivo en la transparencia de las entidades federativas. Aunque no es un tema que ha sido abordado ampliamente en la literatura desde este enfoque, los resultados dan muestra empírica de dichas premisas.
\end{abstract}

PALABRAS CLAVE: Transparencia, Inconformidades, Educación, Gobiernos Subnacionales.

\footnotetext{
1 Doctor en Estudios Fiscales con especialidad en Hacienda Pública. Especialista en materia de transparencia, destacando su participación como Consejero Ciudadano suplente en el órgano garante estatal; Consejero Ciudadano de Transparencia del municipio de Zapopan, así como miembro de organismos de la sociedad civil en materia de transparencia en la que ha sido enlace para la implementación de diversas iniciativas ciudadanas en la materia.
} 
CONTENIDOS: 1. Elementos introductorios. - 2. Determinantes y efectos de la transparencia. - 2.1 La transparencia y sus obstáculos. - 2.2 La transparencia y el nivel educativo. - 3. Metodología. - 4. Análisis de resultados. - 4.1. Estadísticos descriptivos y correlaciones. - 4.2 Transparencia como variable dependiente de las Inconformidades y el Nivel educativo (determinantes). 5. Conclusiones. - Bibliografia.

\section{Education level and revision appeals as determinants in the subnational government's transparency in México}

ABSTRACT: This article is intended to answer the questions related to the link between the nonconformities and the educational level in the subnational governments' transparency in Mexico. Throughout the article, readers may understand the conceptual and theoretical elements that support this approach. The first outcome induces negative results about the nonconformities in governmental transparency, while the second one explains that as higher the school level in citizens, transparency levels increase. The article's results are not conclusive but bring an empiric sample to develop frameworks to test the correlation between the educational level and the nonconformities with the transparency.

KEYWORDS: Transparency, Revision appeals, Education, Subnational Governments. 


\section{Elementos introductorios}

Como parte de las acciones para la mejora institucional surgidas en la década de los noventa, la transparencia y la rendición de cuentas surgen como conceptos y elementos que han transformado la manera en que se gestiona lo público. La relevancia de la transparencia a lo largo de las últimas tres décadas a nivel internacional es producto, principalmente, de las experiencias económicas como la crisis mexicana de 1994 y la crisis asiática de 1997 (Philips y Stewart, 2008), posterior a ello, instituciones internacionales centraron su interés a las relaciones entre el buen gobierno y la transparencia, bajo la premisa de lograr mejores resultados económicos y sociales (De Renzio y Wehner, 2015). El buen gobierno, es la antítesis, prevención y remedio ante la aparición de la corrupción política y administrativa, contribuyendo notablemente la exigencia de la transparencia (Canales y RomeroTarin, 2017), en este sentido, la transparencia tiene un papel instrumental como medio para facilitar el control de los poderes públicos, la participación en la toma de decisiones y la colaboración de la sociedad con el Estado en la acción del Gobierno (Delgado, 2014) $)^{2}$.

La transparencia es un elemento conectado y necesario para que exista rendición de cuentas, pero, además, se le asignan una serie de funciones como la mejora de la eficacia y eficiencia de la gestión pública o la prevención de la corrupción (VilloriaMendieta, 2014), porque a través de la transparencia los ciudadanos pueden ejercitar un escrutinio más cualificado de la acción de gobierno (Sierra-Rodríguez J., 2020)

La transparencia puede ser conceptualizada como el resultado de las prácticas que en materia de acceso a la información realizan las entidades públicas, tomando como base la obligación de difundir información y mejorar sus canales de acceso a la misma (Mora-Pérez, 2020), por lo que un gobierno puede considerarse transparente si genera las condiciones de acceso a la información a la población, pone a disposición de la misma la mayor cantidad de información que genere o posea, con la mayor calidad posible (Mora-Pérez, 2020). Por su parte, Delgado (2015) define a la transparencia como una cualidad, derivada de la satisfacción por parte de las administraciones públicas a la demanda de información ciudadana.

En principio, es importante establecer la relación entre los conceptos de transparencia, acceso a la información y rendición de cuentas, a partir de la propia información pública. La transparencia y el acceso a la información tienen un vínculo estrecho a partir de una noción más amplia: el derecho a la libertad de opinión y de expresión. Es a partir de este derecho que subyace la premisa de que las personas son libres para investigar, solicitar y recibir cualquier tipo de información de las instituciones públicas.

${ }^{2}$ Citado en: Sierra-Rodríguez, Javier (2020) Estado de alarma, transparencia gubernamental y rendición de cuentas durante la crisis del COVID-19. 
Delgado (2015) establece que la transparencia y el derecho de acceso a la información tienen una clara vinculación, pero no son lo mismo. Al ser una garantía constitucional, la transparencia forma parte del derecho de acceso a la información, ya que se establece como obligación de las instituciones gubernamentales el poner a disposición de la población información de carácter público (INEGI, 2017). El derecho de acceso a la información pública garantiza la transparencia, pero es una acción previa (Delgado, 2015).

A partir de esta obligación legal y derivado de las diversas acciones que se ha realizado para fortalecer la transparencia gubernamental, ha sido posible analizar este mecanismo, al menos, desde tres enfoques: ${ }^{3}$ un enfoque normativo, un enfoque económico y un enfoque institucional. Es a partir de este último enfoque que se toma como base para el desarrollo de este documento, principalmente por el efecto de la transparencia en la gestión institucional, en el cumplimiento de sus obligaciones y en la evaluación de la aplicación de los recursos públicos, entre otros, lo que se ha demostrado en la literatura revisada.

Por su parte, la rendición de cuentas puede ser conceptualizada como el mecanismo mediante el cual las autoridades se someten a la evaluación de su desempeño, a la opinión pública sobre estos resultados y a las propias sanciones que puedan generarse por el incumplimiento a sus obligaciones (Mora-Pérez, 2020). La relación de la transparencia con la rendición de cuentas reside en la visibilización de la acción pública que es del conocimiento y escrutinio público.

Como se ha comentado anteriormente, el análisis de la transparencia desde un enfoque institucional ha permitido establecer su evolución de manera gradual, ${ }^{4}$ principalmente en el acceso y disponibilidad de la información pública, pudiendo asociar esta evolución con el desarrollo de los propios modelos de gestión pública, a partir del enfoque sistémico de las organizaciones. En este sentido, el acceso y la disponibilidad de información pública se puede sugerir en cuatro momentos (MoraPérez, 2020).

\footnotetext{
${ }^{3}$ De acuerdo con el autor, en el enfoque normativo se analiza a la transparencia como el mecanismo que garantiza el derecho de acceso a la información de los individuos, el cumplimiento de las disposiciones normativas y la evolución de estas. Desde un enfoque económico se analiza a la transparencia por su impacto en el crecimiento económico a través del uso de la información, la cual, entre otros aspectos, reduce la incertidumbre y promueve la eficiencia en las decisiones económicas. Desde un enfoque institucional se analiza a la transparencia por su efecto en la gestión pública, en las instituciones a través de la mejora del desempeño, la diminución de la corrupción, la rendición de cuentas y la eficiencia en la aplicación de los recursos públicos. Consultar en: Mora-Pérez (2020).

4 Esta clasificación es realizada por el autor, de acuerdo con lo establecido en la norma como transparencia proactiva, transparencia focalizada y a las nuevas tendencias en materia de gobierno abierto. Se parte del concepto de información de segunda generación establecido por la OEA para la transparencia focalizada (ver: http://www.oas.org/es/sap/dgpe/ACCESO/Derecho.asp)
} 


\section{Gráfico 1. Evolución de la transparencia en la publicación de la información}

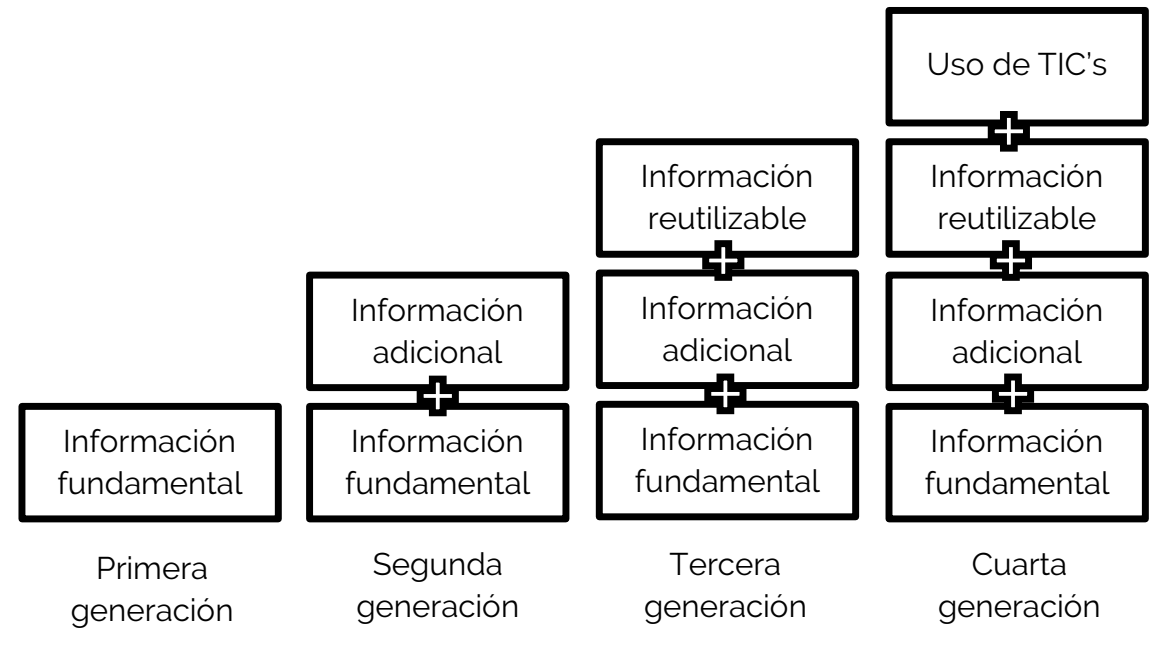

Fuente: Mora-Pérez (2020).

La base de la transparencia se establece con cuatro pilares complementarios (Fierro y Gil-Garcia, 2011): disponibilidad de información; calidad y utilidad de la información; uso intensivo de tecnologías de información y comunicación, así como estrategias y herramientas de participación y colaboración. La disponibilidad se refiere a la información que proveen las instituciones públicas sin que la ciudadanía tenga que pedirla; la calidad y utilidad aluden a la posibilidad de que la información sea usada de forma significativa por quien la solicite; el tercer pilar facilita el acceso a la información pública; y el cuarto, busca promover una mayor participación ciudadana a través de canales y medios de colaboración entre los distintos actores del sistema (Fierro y Gil-García, 2011).

La transparencia no es solo información, comunicación y documentación administrativa claras y fiables, implica la facilidad de su accesibilidad y de su receptividad (Canales y Romero-Tarín, 2017), estos elementos deben ser garantizados por las instituciones para garantizar el ejercicio del derecho de acceso a la información. Las herramientas en las que se basa la transparencia para la obtención de información pública aparecen en la TABLA 1. 
Tabla 1. Herramientas de la transparencia

\begin{tabular}{|c|c|}
\hline Herramienta & Concepto \\
\hline $\begin{array}{l}\text { La transparencia } \\
\text { activa }\end{array}$ & $\begin{array}{l}\text { Se refiere a la publicación de información fundamental a } \\
\text { que están obligados los entes públicos de acuerdo con } \\
\text { disposiciones normativas y que deberá estar disponible sin } \\
\text { que medie solicitud alguna. }\end{array}$ \\
\hline $\begin{array}{l}\text { La transparencia } \\
\text { proactiva }\end{array}$ & $\begin{array}{l}\text { Se refiere a la publicación de información adicional a la que } \\
\text { se establece como mínimo en los ordenamientos y que es } \\
\text { relativa a casos de especial interés público. En sentido } \\
\text { estricto, este mecanismo permite incrementar la oferta de } \\
\text { información pública de acuerdo con la demanda de los } \\
\text { usuarios. }\end{array}$ \\
\hline $\begin{array}{l}\text { La transparencia } \\
\text { reactiva }\end{array}$ & $\begin{array}{l}\text { Se refiere al procedimiento de acceso a la información que } \\
\text { propicia la entrega de información pública de libre acceso } \\
\text { a los ciudadanos solicitada mediante una solicitud de } \\
\text { información, la cual no es información fundamental. }\end{array}$ \\
\hline $\begin{array}{l}\text { La transparencia } \\
\text { focalizada }\end{array}$ & $\begin{array}{l}\text { Es información específica y útil para facilitar la toma de } \\
\text { decisiones en distintas temáticas que tienen incidencia en } \\
\text { la vida cotidiana de un grupo amplio pero definido de la } \\
\text { sociedad. Las políticas de transparencia focalizada se } \\
\text { estructuran para generar información estandarizada, } \\
\text { comparable y desagregada con un propósito público } \\
\text { especifico como la evaluación de las políticas públicas. }\end{array}$ \\
\hline $\begin{array}{l}\text { La transparencia } \\
\text { digital } 5^{5}\end{array}$ & $\begin{array}{l}\text { Es información fundamental, proactiva o focalizada que se } \\
\text { desarrolla para un segmento general o particular de la } \\
\text { población a partir del uso de tecnologias emergentes que } \\
\text { faciliten de manera innovadora su acceso, respuesta y que } \\
\text { fomenten la cultura de la transparencia para el } \\
\text { fortalecimiento del derecho de acceso a la información. }\end{array}$ \\
\hline
\end{tabular}

Fuente: elaboración propia con datos de Mora-Pérez (2020).

En este orden de ideas necesario considerar lo establecido por Sánchez de DiegoFernández de la Riva y Sierra-Rodríguez (2020) respecto a los retos que tiene la transparencia activa e incentivar que se vaya más allá de lo que establece la regulación (transparencia proactiva y focalizada), planteándose las siguientes interrogantes sobre el contenido a publicar (qué publicar), sobre la manera de publicar (cómo publicar), con el momento y la vigencia de la información (cuándo publicar) y con el lugar de publicación (dónde publicar), aunque un problema recurrentemente es la falta de control sobre la transparencia activa (Sanchez de Diego-Fernandez de la Riva y Sierra-Rodriguez, 2020).

\footnotetext{
${ }^{5}$ Este concepto surge como una respuesta innovadora a las políticas de acceso a la información a través de las tecnologias emergentes. Se propone para futuras investigaciones.

${ }^{6}$ Este concepto cobra relevancia al ser el medio electrónico una forma de comunicación en materia de transparencia.
} 
En México, el derecho de acceso a la información pública se consagró con la reforma del artículo $6^{\circ}$ constitucional en 1977, cuando se anexó la expresión "el derecho a la información será garantizado por el Estado"; no obstante, su concepción era ambigua y carecía de un procedimiento definido para ejercer este derecho (INEGI, 2017). Por ello, la reforma de 2007 al artículo $6^{\circ}$ Constitucional estableció los principios, condiciones y exigencias minimas que deberian cumplir las distintas legislaciones del país y cada nivel de gobierno, además de obligar a los poderes públicos a transparentar la información sobre su quehacer gubernamental (INEGI, 2017)

Siguiendo con las reformas en la materia, en 2014, se reformaron diez artículos constitucionales con la intención de definir un sistema integral y coherente en materia de transparencia y cuyos ejes principales fueron el dotar de autonomía a los organismos garantes del derecho de acceso a la información a nivel estatal y federal, el ampliar las atribuciones y responsabilidades del organismo garante federal y por último el definir y aumentar el número de instituciones obligadas a publicar información sobre sus funciones y sobre el uso de fondos públicos, (INEGl, 2017).

Como se observa, en México las reformas en materia de acceso a la información han fortalecido el ejercicio de este derecho de manera progresiva. En principio, con la solidez de los marcos normativos, tanto en lo general como en lo local, además, las instituciones públicas (definidas por la norma como "sujetos obligados") han desarrollado diversas estrategias enfocadas a mejorar sus prácticas de acceso y disponibilidad de información, principalmente con la Plataforma Nacional de Transparencia y los propios portales de transparencia de cada sujeto obligado.

El planteamiento de este documento tiene como finalidad, en principio, conocer la relación de los recursos de revisión (inconformidades) en las solicitudes de acceso a la información, bajo la premisa de que los sujetos obligados han encontrado en las respuestas negativas, incompletas o inexactas un medio que puede desincentivar las solicitudes de información y que no contribuye a la disponibilidad, calidad y utilidad de la información, pilares indispensables establecidos por Fierro y Gil-García (2011).

Además, se plantea conocer la relación que tiene el nivel educativo con la transparencia, partiendo del supuesto de que una sociedad con mayor nivel educativo tiene acceso a más información y desarrolla mayor conciencia para el ejercicio de sus derechos, así como su interés en conocer y evaluar el quehacer gubernamental, a la vez, sus habilidades para atraerse de información por medios digitales pueden ser mayores, por lo que el acceso a la información se incrementa. En la revisión de la literatura se establece que el nivel educativo está asociado con niveles de desarrollo de habilidades tecnológicas, lo que resulta relevante al ser el medio digital por el que se realizan más solicitudes de acceso a la información.

El análisis de los elementos anteriores se realiza de manera descriptiva para las 32 entidades federativas de México, con base en indicadores oficiales y relacionando de manera empírica las variables determinadas para cada planteamiento. 


\section{Determinantes y efectos de la transparencia}

La literatura existente sobre la transparencia puede dividirse en dos principales grupos de análisis. En principio, los estudios sobre los determinantes de la transparencia, seguidos por los estudios sobre los efectos de la transparencia, ambos grupos de estudios y análisis han aportado resultados que han permitido construir un marco teórico y empírico cada vez más robusto.

Entre los estudios sobre determinantes de la transparencia se destacan principalmente los realizados por Tavares y Ferreira (2014) y Zuccolotto y Teixeira (2014) que muestran una relación significativa entre la educación y el nivel de transparencia (proactiva y focalizada), además del estudio de Fox, Haight, y PalmerRubin (2011) que analiza los recursos de revisión asociados a la transparencia. En la revisión de la literatura se pueden destacar otros estudios basados en determinantes como: Guillamon, Bastida y Benito (2011) enfocado a la estructura del gobierno, o bien, el estudio realizado por De Araujo y Tejero (2016) en donde se incluye el género en niveles ejecutivos, ambos con variables de tipo institucional.

Para efecto de este documento se toma como base los estudios de Tavares y Ferreira (2014) y Zuccolotto y Teixeira (2014) para el caso del nivel educativo y de Fox, Haight, y Palmer-Rubin (2011) para el caso de los recursos de revisión (inconformidades).

En cuanto a los efectos de la transparencia, la literatura refiere a los estudios de Alt y Lassen (2006) y Andreula y Chong (2015) como un medio fundamental para mejorar los acuerdos de gobernanza pública, además del modelo de la OCDE (2005) sobre el Buen Gobierno en donde se establecía a la transparencia y la rendición de cuentas como elementos promovidos por el acceso a la información pública. Alt, Lassen y Rose (2006) y Heald (2013) sostienen que la transparencia puede garantizar la legitimidad del gobierno y la eficiencia de los servicios públicos. Por su parte, la transparencia se considera como un mecanismo para el buen gobierno (De Renzio y Wehner, 2015).

En estudios como Teig (2009), Hammed (2005) y Bastida y Benito (2007) se relacionan los hallazgos de la transparencia (proactiva y focalizada) con el combate a la corrupción y el desarrollo de instituciones más fuertes. Además, Drabek y Payne (2001), Gelos y Wei (2002), Shin y Glennerster (2003), Bernoth y Wolff (2008) coinciden en que hay menor riesgo de que los políticos puedan hacer negocio al existir mayores niveles de transparencia (proactiva y focalizada).

Como se observa en los estudios sobre los efectos de la transparencia, se asocian principalmente al buen gobierno y el combate a la corrupción, conceptos relacionados con la rendición de cuentas, aunque para este documento se establecen como parte de la revisión de la literatura los efectos de la transparencia, solo se considerarán los determinantes de esta, tomando como elementos los recursos de revisión y el nivel educativo. 


\subsection{La transparencia y sus obstáculos}

La expansión de los medios electrónicos y el internet ha propiciado que las instituciones públicas pongan a disposición de la sociedad una amplia cantidad de información a través de sus portales, sin embargo, el acceso a la misma se realiza principalmente a través de la consulta a enlaces y en formatos rígidos. Como lo establece Sierra-Rodríguez (2018), llama la atención la cantidad de criterios que debe regir la publicación de la información que sea estructurada de forma fácil, sencilla y comprensible, además de las dificultades técnicas para cumplir la totalidad de las especificaciones. Con la ley de transparencia ya no solo importa la información que se publica, sino cómo se publica (Sierra-Rodríguez, 2018).

El uso de los medios electrónicos se ha convertido en una de las opciones preferidas por los solicitantes para realizar solicitudes de información y, por ende, para recibir respuestas a las mismas. Como lo establecen Fierro y Gil-García (2011) la disponibilidad de información, su utilidad y el uso intensivo de tecnologías de información son pilares fundamentales de la transparencia. La implementación de mecanismos que faciliten el acceso a la información permite transitar hacia sociedades mejor informadas que puedan ser partícipes de la toma de decisiones públicas, lo que involucra otro de los pilares de la transparencia plateados por Fierro y Gil-García (2011), la participación ciudadana.

La participación de la sociedad en el acceso a la información se puede evidencias a partir de las solicitudes de información realizadas. En México, existe un incremento del $453 \%$ de incremento en las solicitudes de transparencia en el periodo 2008 al 2019, de acuerdo con los datos del Sistema Nacional de Transparencia ${ }^{7}$. Además, en este mismo periodo, se observa un incremento del 1,516\% en la cantidad acumulada de recursos de revisión ${ }^{8}$ ante los órganos garantes por la falta de respuesta o la inconformidad por la respuesta recibida por parte de las autoridades.

Cejudo y Torres $^{9}$ manifiestan que debido a que muy poca gente toma el paso adicional de manifestar su inconformidad con la respuesta recibida, es posible que $\begin{array}{llll}7 & \text { Consultar en: Estadisticas del Sistema Nacional de Transparencia } \\ \text { (http://Www.snt.org.mx/index.php/informacioninteres/estadisticas) }\end{array}$

8 De acuerdo con el artículo 143 de la Ley General de Transparencia y Acceso a la Información Pública de México los recursos de revisión son un recurso administrativo del solicitante que procederá en contra de determinaciones de los sujetos obligados como la clasificación de la información; la declaración de inexistencia de información; la entrega de información incompleta; la entrega de información que no corresponda con lo solicitado; la falta de respuesta a una solicitud de información dentro de los plazos establecidos en la ley; la notificación, entrega o puesta a disposición de información en una modalidad o formato distinto al solicitado; la entrega o puesta a disposición de información en un formato incomprensible y/o no accesible para el solicitante; los costos o tiempos de entrega de la información; la falta de trámite a una solicitud; entre otros.

9 Artículo de opinión consultado en 2020 publicado en el portal del medio digital Animal Político y titulado El problema de las "inexistencias" en el acceso a la información, publicado en: https://www.animalpolitico.com/intuiciones-y-evidencia/el-problema-de-las-inexistencias-en-elacceso-a-la-informacion/ 
haya miles de solicitantes que no reciben la información que piden, bajo la justificación de que ésta no existe (cuando en realidad sí existe) lo que limita el acceso a la información.

La incidencia de los recursos de revisión puede ser un medio que obstaculice el derecho a la información, en principio, al haber mayor cantidad de solicitudes se genera mayor carga de trabajo en los sujetos obligados, lo que puede afectar la calidad y la atención en la entrega de información, además de inconformidades por parte de los ciudadanos.

Tomando como referencia lo dicho por Cejudo y Torres, los ciudadanos que se inconforman y realizan el recurso de revisión han incrementado este trámite en los últimos años de acuerdo con las estadísticas del Sistema Nacional de Transparencia de México, lo que puede estar asociado de manera negativa en el acceso a la información derivado del mal estado de los sistemas de gestión de archivos y registros (respuestas parciales o incompletas), plazos importantes (respuesta fuera de tiempo) o tarifas elevadas (costos de reproducción).

Lo anterior, ha quedado en evidencia en la última versión disponible de la Medición de Transparencia y Acceso a la Información en México del CIDE (2014), que observan esas y otras situaciones que obstaculizan el derecho de acceso a la información por los sujetos obligados (véase TABLA 2).

Como se observa en la tabla, las principales situaciones encontradas se asocian a los mecanismos de transparencia activa y reactiva, por lo que es necesario acentuar la observación en estos mecanismos para evitar los recursos de revisión.

Además del aspecto normativo, Fox, Haight, y Palmer-Rubin (2011) consideran al recurso de revisión como un procedimiento de queja o inconformidad ciudadana respecto a una negativa injustificada o una respuesta insuficiente, derivado principalmente en que los sujetos obligados están negando cada vez más la "existencia" de la información solicitada.

A partir de lo anterior, se infiere en este documento que los recursos de revisión (considerados como inconformidades) tienen una relación negativa en el acceso a la información (solicitudes de transparencia), por lo que se establece que aquellas entidades en las que se tramitan mayores recursos de revisión tienen efectos negativos en sus solicitudes de acceso a la información.

Ante estos obstáculos, es pertinente plantearse el reto de implementar vías de contacto directo, complementarias a las ejercidas mediante el trámite administrativo de solicitud de acceso y de recurso de revisión, buscando el diálogo entre sociedad y gobierno para facilitar el acceso de manera más ágil y sencilla (Sánchez de DiegoFernández de la Riva y Sierra-Rodríguez, 2020). 
Tabla 2. Obstáculos del acceso a la información

\begin{tabular}{|l|c|}
\hline \multicolumn{1}{|c|}{ Situación que genera obstáculo } & $\begin{array}{c}\text { Mecanismo } \\
\text { asociado al } \\
\text { obstáculo }\end{array}$ \\
\hline $\begin{array}{l}\text { El promedio de cumplimiento con la información } \\
\text { fundamental es del 85\%, por lo que los sujetos obligados no } \\
\text { cumplen con la obligación legal de poner a disposición de } \\
\text { los ciudadanos información básica. }\end{array}$ & $\begin{array}{c}\text { Transparencia } \\
\text { activa }\end{array}$ \\
\hline $\begin{array}{l}\text { Conforme a las buenas prácticas de gobierno abierto, es } \\
\text { importante que la información publicada en los portales de } \\
\text { transparencia tenga acceso libre, y que los ciudadanos } \\
\text { puedan reutilizarla sin limitación o gratuitamente se } \\
\text { encontró que solo en 11 entidades tenian alguna } \\
\text { información en formatos abiertos. }\end{array}$ & $\begin{array}{c}\text { Transparencia } \\
\text { activa }\end{array}$ \\
\hline $\begin{array}{l}\text { En promedio, en México, un usuario debe esperar 9.55 días } \\
\text { hábiles para recibir información. Este promedio, sin } \\
\text { embargo, disfraza una dispersión interesante, pues existen } \\
\text { casos en donde el sujeto obligado tardó más de veinte días } \\
\text { en proporcionar respuesta. }\end{array}$ & $\begin{array}{c}\text { Transparencia } \\
\text { reactiva }\end{array}$ \\
\hline $\begin{array}{l}\text { Mejorar la comunicación entre el ciudadano y autoridad es } \\
\text { un principio de gobierno electrónico, respecto a los } \\
\text { mecanismos de comunicación para reportar fallas del } \\
\text { portal o sobre la calidad de información en los portales se } \\
\text { tuvo un promedio del 33\%. }\end{array}$ & $\begin{array}{c}\text { Transparencia } \\
\text { activa }\end{array}$ \\
\hline $\begin{array}{l}\text { Respecto a las solicitudes de información, de las 2950 } \\
\text { solicitudes presentadas, 8.24\% (243 solicitudes) no fueron } \\
\text { respondidas; 3.69\% del total (109 solicitudes) tuvieron } \\
\text { prevención como primera respuesta y en 8.34\% de los casos } \\
\text { (246 solicitudes) el sujeto obligado solicitó prórroga. }\end{array}$ & $\begin{array}{c}\text { Transparencia } \\
\text { reactiva }\end{array}$ \\
\hline
\end{tabular}

Fuente: elaboración propia.

\subsection{La transparencia y el nivel educativo}

Como se ha dicho en apartados anteriores, el nivel educativo de una sociedad puede relacionarse con el desarrollo de las habilidades en el uso de las tecnologías de la información. La relación del nivel educativo con el desarrollo de habilidades en el uso de las tecnologías de la información se realiza como un medio de alfabetización, adquisición de conocimientos y habilidades.

De acuerdo a la Encuesta Nacional de Calidad e Impacto Gubernamental 2019 , $\mathrm{ENCIG}^{10}$, solo el 32\% de la población en México ha interactuado con el gobierno a través de internet, el $85 \%$ enfrentó barreras gubernamentales, el 36\% se enfrentó a asimetrias en la información proporcionada y el $6 \%$ problemas con las TIC's. Además, se establece que los medios electrónicos son el medio preferido por el $43 \%$ de los

10 Encuesta Nacional de Calidad e Impacto Gubernamental (ENCIG). Recuperado de: https://www.inegi.org.mx/programas/encig/2019/ 
solicitantes de información, siendo la segunda opción después de la solicitud personal.

En cuanto al nivel educativo, existen diferentes indicadores que miden el rendimiento educativo, en algunos casos se utilizan como indicadores las habilidades en el uso de las tecnologías de la información. La literatura en materia de transparencia ha demostrado que el nivel educativo tiene una relación significativa en la transparencia, con base en los estudios realizados por Tavares y Ferreira (2014) y Zuccolotto y Teixeira (2014).

En México, el nivel educativo que se tiene como obligatorio garantizar por el Estado es la educación media superior ${ }^{11}$. Una de las acciones que contribuye a la disminución de la desigualdad en México es el incremento de los niveles educativos en la sociedad, al menos, en el nivel obligado constitucionalmente, la educación media.

Tomando como referencia el indicador de eficiencia terminal ${ }^{12}$ (como uno de los indicadores de rendimiento educativo) el nivel de educación media para el caso mexicano es del 66,6 por ciento, mientras que en el caso del promedio de escolaridad es de 9,4 años, lo que equivale a un nivel educativo básico (secundaria). De igual manera, se evidencia que el $68 \%$ de la población refiere estar satisfecha con los servicios de educación a nivel nacional ${ }^{13}$ (incluido el nivel medio superior), situación que agrava la calidad de la educación en México.

Es importante mencionar que de acuerdo con la Encuesta Nacional de Acceso a la Información Pública y Protección de Datos Personales 2019, ENAID ${ }^{14}$, en México el perfil sociodemográfico de los solicitantes de información muestra que el $41 \%$ de quienes realizan una solicitud de información tienen nivel educativo superior o posgrado, el 33\% educación media y el 25\% educación básica.

\footnotetext{
${ }^{11}$ Con base en el artículo $3^{\circ}$ primer párrafo de la Constitución Política de los Estados Unidos Mexicanos, el cual ha sido reformado en cuatro ocasiones: 12 de noviembre de 2002, og de febrero de 2012, 29 de enero de 2016 y 15 de mayo de 2019. En la reforma de 2012 se elevó a rango constitucional la obligatoriedad de la educación media superior en México

12 Estadisticas del Sistema Educativo Nacional. Consular en: http://snie.sep.gob.mx/descargas/estadistica_e_indicadores/estadistica_e_indicadores_educativos_ 33Nacional.pdf

13 Con base en los resultados de la Encuesta Nacional de Calidad e Impacto Gubernamental (ENCIG) 2019. Consultar en: https://WwW.inegi.org.mx/contenidos/programas/encig/2019/doc/encig2019_principales_resultad os.pdf

${ }^{14}$ Con base en los resultados de la Encuesta Nacional de Acceso a la Información Pública y Protección de Datos Personales (ENAID). Consultar en: https://Www.inegi.org.mx/programas/enaid/2019/
} 


\section{Gráfico 2. Niveles educativos de los solicitantes de información}

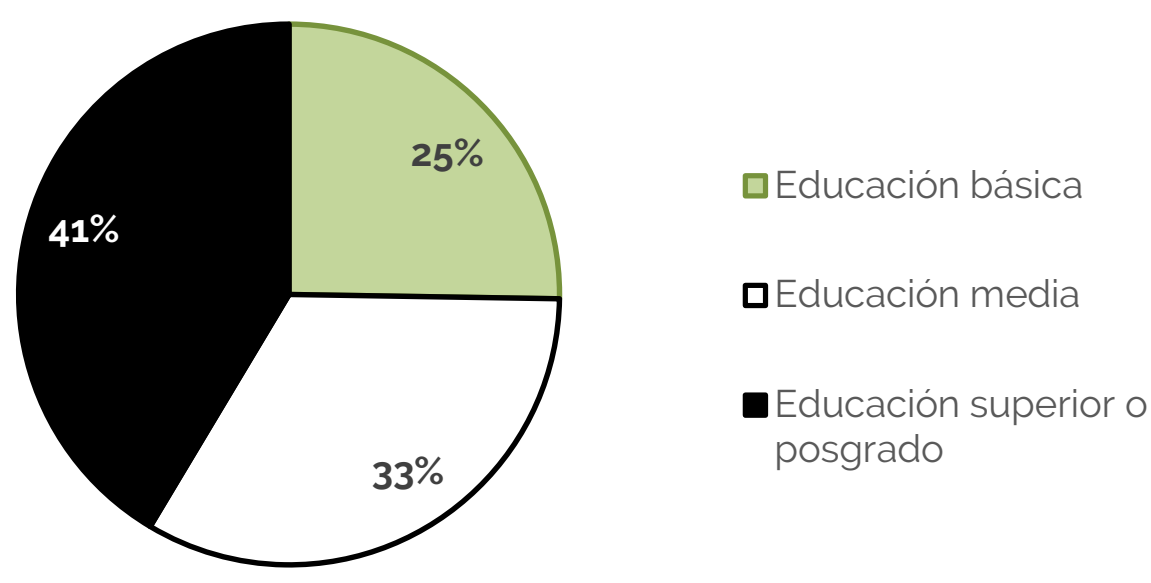

Fuente: elaboración propia con datos del ENAID (2019).

Con base en lo anterior, es posible inferir que, a mayor nivel educativo en la sociedad, mayor será la participación en las solicitudes de información. Por lo que tomando como indicador el nivel de eficiencia terminal en educación media (como el nivel constitucional obligatorio) las entidades que presentan mayores niveles de educación media ejercen de mejor manera el derecho de acceso a la información.

\section{Metodología}

El análisis realizado tiene un corte transversal de la información, se utilizan tres variables de estudio descriptivo para las 32 entidades federativas (gobiernos subnacionales) de México con corte de la información al año 2019. El estudio es de tipo empírico y toma los datos de fuentes oficiales como el Sistema Nacional de Transparencia ${ }^{15}$ y el Sistema Educativo Nacional ${ }^{16}$, los cuales son abiertos a la sociedad como una práctica de transparencia proactiva. Por lo que en este documento se describen e interpretan datos estadisticos oficiales para el análisis.

Las variables que se utilizaron constan en la TABLA 3.

\footnotetext{
${ }^{15}$ Consultar en: http://www.snt.org.mx/index.php/informacioninteres/estadisticas

${ }^{16}$ Consultar en: https://www.inee.edu.mx/evaluaciones/bases-de-datos/
} 
Tabla 3. Variables e indicadores para utilizar en la investigación

\begin{tabular}{|c|c|c|c|c|}
\hline Variable & Indicador & Sentido & Tipo & Fuente \\
\hline Transparencia & $\begin{array}{l}\text { Solicitudes de acceso a } \\
\text { información } \\
\text { reactiva) }\end{array}$ & & Dependiente & $\begin{array}{l}\text { Sistema Nacional } \\
\text { de Transparencia }\end{array}$ \\
\hline Inconformidades & $\begin{array}{l}\text { Recursos de revisión } \\
\text { interpuestos ante los } \\
\text { órganos garantes }\end{array}$ & - & Independiente & $\begin{array}{l}\text { Sistema Nacional } \\
\text { de Transparencia }\end{array}$ \\
\hline Nivel educativo & $\begin{array}{l}\text { Eficiencia terminal en } \\
\text { educación media }\end{array}$ & + & Independiente & $\begin{array}{l}\text { Sistema Educativo } \\
\text { Nacional }\end{array}$ \\
\hline
\end{tabular}
Fuente: Elaboración propia.

En la siguiente figura se muestran las relaciones entre las variables analizadas (GRÁFICO 3).

\section{Gráfico 3. Modelo de relación de variables}

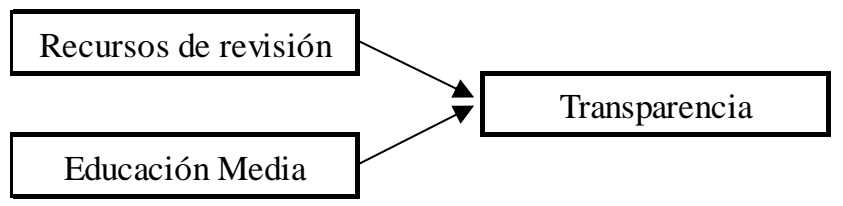

Fuente: Elaboración propia.

Tabla 4. Matriz de congruencia de la investigación

\begin{tabular}{|c|c|c|c|c|}
\hline $\begin{array}{c}\text { Objetivo de } \\
\text { investigación }\end{array}$ & $\begin{array}{l}\text { Preguntas de } \\
\text { investigación }\end{array}$ & Variable & Indicadores & Hipótesis \\
\hline \multirow{2}{*}{$\begin{array}{c}\text { Determinar el } \\
\text { efecto de las } \\
\text { inconformidades } \\
\text { y el nivel } \\
\text { educativo en la } \\
\text { transparencia de } \\
\text { las entidades } \\
\text { federativas. }\end{array}$} & $\begin{array}{c}\text { ¿Cuál es el } \\
\text { efecto de las } \\
\text { inconformidade } \\
\text { s en la } \\
\text { transparencia? }\end{array}$ & Inconformidades & $\begin{array}{l}\text { Recursos de } \\
\text { revisión }\end{array}$ & $\begin{array}{l}\text { H1: La variable } \\
\text { Inconformidades } \\
\text { tiene una } \\
\text { relación negativa } \\
\text { con la } \\
\text { Transparencia }\end{array}$ \\
\hline & $\begin{array}{l}\text { ¿Cuál es el } \\
\text { efecto del nivel } \\
\text { educativo en la } \\
\text { transparencia? }\end{array}$ & Nivel educativo & $\begin{array}{c}\text { Eficiencia } \\
\text { terminal en } \\
\text { educación } \\
\text { media }\end{array}$ & $\begin{array}{l}\text { H2: La variable } \\
\text { Nivel educativo } \\
\text { tiene una } \\
\text { relación positiva } \\
\text { con la } \\
\text { Transparencia }\end{array}$ \\
\hline
\end{tabular}

Fuente: elaboración propia. 


\section{Tabla 5. Hipótesis de la investigación}

\begin{tabular}{|c|}
\hline \multicolumn{1}{|c|}{ Descripción de las hipótesis } \\
\hline H1. A mayores Inconformidades, menor Transparencia. \\
\hline H2. A mayor Nivel educativo, mayor Transparencia. \\
\hline Fuente: Elaboración propia.
\end{tabular}

Esta investigación se plantea como una aportación más para el debate académico sobre las variables que se relacionan con la transparencia en las entidades federativas de México. Una vez finalizada la fase de estructuración de datos, se procedió a la codificación de los indicadores utilizando el paquete estadístico SPSS (Statistical Package for Social Sciences).

Con el objeto de depurar la base de datos y detectar posibles errores en la trascripción de estos, se utilizaron técnicas descriptivas, calculándose la distribución de frecuencias de cada variable para comprobar que todos los códigos de las variables se situaban entre los valores posibles. Se realizó el análisis para conocer el efecto de las dos variables consideradas como determinantes (Inconformidades y Nivel educativo) y su efecto en la variable Transparencia.

\section{Análisis de resultados}

\subsection{Estadísticos descriptivos y correlaciones}

En principio, se muestran los resultados con los estadisticos descriptivos con la finalidad de verificar que la totalidad de información se hubiese analizado. Encontramos la totalidad de los datos para las 32 entidades federativas (TABLA 6).

Tabla 6. Estadísticos descriptivos del Modelo

\begin{tabular}{|l|r|r|r|}
\hline & \multicolumn{1}{|c|}{ Media } & $\begin{array}{c}\text { Desviación } \\
\text { estándar }\end{array}$ & $\mathrm{N}$ \\
\hline Transparencia & 13,1256 & 18,83763 & 32 \\
Inconformidades & 89,3919 & 19,88855 & 32 \\
Nivel educativo & 41,5841 & 18,82138 & 32 \\
\hline
\end{tabular}

Fuente: Elaboración propia.

Posteriormente, se realiza un análisis a través del coeficiente de correlaciones con la finalidad de analizar las relaciones entre las variables. Los resultados muestran correlaciones significativas entre las variables y en el sentido que se propone para cada una (TABLA 7). 
Tabla 7. Correlación de variables del Modelo

\begin{tabular}{|c|c|c|c|c|}
\hline & & Transparencia & Inconformidades & $\begin{array}{c}\text { Nivel } \\
\text { educativo }\end{array}$ \\
\hline \multirow{3}{*}{ Transparencia } & Correlación de Pearson & 1 & -.516 & $.505^{\prime \prime}$ \\
\hline & Sig. (bilateral) & & .003 &, 003 \\
\hline & $\mathrm{N}$ & 32 & 32 & 32 \\
\hline \multirow{3}{*}{ Inconformidades } & Correlación de Pearson & -.516 & 1 & \\
\hline & Sig. (bilateral) & .003 & & \\
\hline & $\mathrm{N}$ & 32 & 32 & \\
\hline \multirow{2}{*}{ Nivel educativo } & Correlación de Pearson & .505 & & 1 \\
\hline & N & $\begin{array}{c}.003 \\
32\end{array}$ & & 32 \\
\hline
\end{tabular}

Fuente: Elaboración propia.

Una vez realizado el ejercicio anterior, se aplicará un análisis tomando el siguiente modelo de regresión:

\section{Ecuación 1.Modelo de regresión utilizado}

$$
Y^{\prime}=a+b_{1} X_{1}+b_{2} X_{2}
$$

\subsection{Transparencia como variable dependiente de las Inconformidades y el Nivel educativo (determinantes)}

El primer análisis del modelo indica que las dos variables introducidas fueron analizadas (TABLA 8)

Tabla 8. Entrada de variable del primer modelo de regresión

Variables entradas/eliminadas ${ }^{a}$

\begin{tabular}{|c|l|l|c|}
\hline Modelo & Variables introducidas & Variables eliminadas & Método \\
\hline 1 & $\begin{array}{l}\text { Nivel educativo, } \\
\text { Inconformidades }\end{array}$ & & Intro \\
\hline
\end{tabular}

a. Variable dependiente: Transparencia

b. Todas las variables solicitadas introducidas.

Fuente: Elaboración propia.

La tabla siguiente proporciona información sobre la capacidad de la línea de regresión para explicar la variación total en la variable dependiente. Como se puede observar, el valor de la $\mathrm{R}^{2}$ es 0,455, lo que significa que un porcentaje considerable de la variación total ha sido explicada. Además, este resumen de modelos indica si las variables son relevantes para el modelo de regresión (TABLA 9) 
Tabla 9. Resumen del primer modelo de regresión

\begin{tabular}{|c|c|c|c|c|}
\hline \multicolumn{5}{|c|}{ Resumen del modelo } \\
\hline $\begin{array}{c}\text { Model } \\
0\end{array}$ & $\mathrm{R}$ & $\begin{array}{c}\mathrm{R} \\
\text { cuadrado }\end{array}$ & $\begin{array}{l}\text { R cuadrado } \\
\text { ajustado }\end{array}$ & $\begin{array}{c}\text { Error } \\
\text { estándar de } \\
\text { la estimación }\end{array}$ \\
\hline 1 & $675^{a}$ & .455 & 418 & 14,37361 \\
\hline
\end{tabular}

En la siguiente tabla, la ANOVA demuestra que el modelo de regresión es estadisticamente significativo al menos en un nivel de 99\%. En consecuencia, se rechaza la hipótesis nula en todos los parámetros estimados que son iguales a cero (TABLA 10).

Tabla 10. Resultados de la ANOVA del primer modelo de regresión

\begin{tabular}{|c|c|c|c|c|c|c|}
\hline \multicolumn{7}{|c|}{ ANOVA $^{\mathrm{a}}$} \\
\hline & & $\begin{array}{l}\text { Suma de } \\
\text { cuadrados }\end{array}$ & gl & $\begin{array}{c}\text { Media } \\
\text { cuadrática }\end{array}$ & $\mathrm{F}$ & Sig. \\
\hline \multirow[t]{3}{*}{1} & Regresión & 5009,127 & 2 & \multirow{3}{*}{$\begin{array}{r}2504,563 \\
206,601\end{array}$} & \multirow[t]{3}{*}{12,123} &, $000^{b}$ \\
\hline & Residuo & 5991,423 & 29 & & & \\
\hline & Total & 11000,550 & 31 & & & \\
\hline
\end{tabular}

a. Variable dependiente: Transparencia

b. Predictores: (Constante), Nivel educativo, Inconformidades Fuente: Elaboración propia.

Basándose en los resultados de la tabla anterior, los coeficientes de las variables más significativas son mostrados en la siguiente TABLA 11. De acuerdo con los resultados mostrados, todos los coeficientes de las variables "Inconformidades" y "Nivel educativo" son estadísticamente significativos, porque los valores T (en valor absoluto) están por encima de 1,96. Del mismo modo, las variables independientes tienen valores de significancia menores que 0.05 por lo que son aceptados.

Tabla 11. Coeficientes del modelo de regresión

\begin{tabular}{|c|c|c|c|c|c|}
\hline \multicolumn{6}{|c|}{ Coeficientes $^{a}$} \\
\hline \multirow{2}{*}{ Modelo } & \multicolumn{2}{|c|}{$\begin{array}{l}\text { Coeficientes no } \\
\text { estandarizados }\end{array}$} & \multirow{2}{*}{\begin{tabular}{|c|}
$\begin{array}{c}\text { Coeficientes } \\
\text { estandarizad } \\
\text { os }\end{array}$ \\
Beta \\
\end{tabular}} & \multirow[b]{2}{*}{$\mathrm{t}$} & \multirow[b]{2}{*}{ Sig. } \\
\hline & B & $\begin{array}{c}\text { Error } \\
\text { estándar }\end{array}$ & & & \\
\hline (Constante) & 33,145 & 14,024 & & 2,363 & .025 \\
\hline $\begin{array}{l}\text { Inconformidad } \\
\text { es }\end{array}$ &,- 429 & 131 &,- 453 & $-3,267$ & .003 \\
\hline $\begin{array}{l}\text { Nivel } \\
\text { educativo }\end{array}$ & .440 & 139 &, 440 & 3,174 &, 004 \\
\hline
\end{tabular}

a. Variable dependiente: Transparencia

Fuente: Elaboración propia. 
Mediante los resultados estadísticos obtenidos, la variable "Inconformidades" muestra una relación inversa con la variable dependiente "Transparencia" mientras que la relación es directa para la variable "Nivel educativo". Al analizar las variables independientes, se puede determinar que el incremento en los recursos de revisión es un obstáculo para la transparencia, por su parte, a mayor población con nivel medio educativo concluido se considera un factor para potenciar la transparencia.

Tabla 5. Cumplimiento de hipótesis a partir de los resultados

\begin{tabular}{|c|l|c|}
\hline \multicolumn{1}{|c|}{ Variable } & \multicolumn{1}{|c|}{ Hipótesis } & Resultado \\
\hline Inconformidades & $\begin{array}{l}\mathrm{H} 1 . \text { A mayores Inconformidades, menor } \\
\text { Transparencia. }\end{array}$ & Se cumple \\
\hline Nivel educativo & $\begin{array}{l}\mathrm{H} 2 . \text { A mayor Nivel educativo, mayor } \\
\text { Transparencia. }\end{array}$ & Se cumple \\
\hline
\end{tabular}

Fuente: elaboración propia.

Los resultados obtenidos en este apartado para las 32 entidades federativas de México muestran que las "Inconformidades" tienen una relación negativa con la "Transparencia", a su vez, el "Nivel educativo" tiene una relación positiva con la "Transparencia", por lo que los resultados de esta investigación comprueban las hipótesis planteadas. Es importante mencionar que, aunque existen estudios que establecen que los valores de los coeficientes de determinación para los modelos de tipo económico-administrativo deben ser superior a 60\% (Estrada y VargasHernández, 2018) se toma en consideración la alta sensibilidad del modelo al solo incluir dos variables independientes.

\section{Conclusiones}

Es importante mencionar que las entidades gubernamentales han puesto interés en las prácticas de transparencia proactiva, a poner a disposición de la población información adicional a la establecida en las obligaciones de transparencia.

En cuanto a los resultados, se concluye que existe una relación negativa (correlación negativa en -0,45) entre las inconformidades y las solicitudes de información realizadas. Esto permite confirmar lo establecido por Cejudo y Torres ${ }^{17}$ respecto a que los recursos de revisión se consideran un obstáculo para el acceso a la información, toda vez que no se garantizan los principios de transparencia, accesibilidad y máxima publicidad establecidos en la Ley General de Transparencia y Acceso a la Información Pública, generando que los solicitantes tengan que realizar de manera complementaria a su solicitud, el procedimiento de inconformidad o queja ciudadana (Fox, Haight, y Palmer-Rubin, 2011). El incremento de estas inconformidades debería ser materia de análisis y seguimiento por parte de los órganos garantes, con la finalidad de mejorar la entrega de información como parte

17 Consultar en: https://www.animalpolitico.com/intuiciones-y-evidencia/el-problema-de-lasinexistencias-en-el-acceso-a-la-informacion/ 
de las funciones y atribuciones de los sujetos obligados. Basado en los principios de rendición de cuentas, los órganos garantes deberían ejercer su atribución de sancionar a los sujetos obligados ante este incumplimiento.

En cuanto al nivel educativo, se confirma de igual manera lo establecido por Tavares y Ferreira (2014) y Zuccolotto y Teixeira (2014) al encontrarse una relación positiva del nivel educativo respecto a la transparencia (correlación de 0,44). Esto permite concluir que las entidades con mejor nivel de eficiencia terminal en educación media tienen mayores niveles de transparencia. Como se estableció en la revisión de la literatura, un mayor grado educativo tiene un efecto positivo en el desarrollo de habilidades en las tecnologias de la información, lo que permite que se ejerza este derecho a través de medios electrónicos. En este punto es importante que se fortalezcan las acciones que fomenten la cultura de la transparencia en niveles educativos precedentes, con la finalidad de que se conozca, se ejerza y se demuestre la utilidad social del derecho de acceso a la información en la sociedad. En la medida que las inconformidades disminuyan y se incremente el nivel educativo (y con ello las habilidades digitales) se fortalecerá la transparencia a través del ejercicio del derecho a la información. Garantizar el derecho de acceso es el supuesto necesario para tener una ciudadanía informada y, en consecuencia, más participativa (Delgado, 2015).

Este documento aporta elementos para el análisis de la transparencia desde enfoques de estudios que no han sido considerados de manera más amplia. La aplicación del estudio en las entidades federativas aporta a la reflexión y el análisis académico de este objeto de estudio. Las limitaciones que se presentan son principalmente la temporalidad de los datos, este estudio es de corte transversal, pero será recomendable realizar análisis de series de tiempo para conocer los cambios en las variables. Además, se construyen variables como determinantes con indicadores que no han sido validados empíricamente de manera más amplia en otros estudios, pero se pueden complementar otros indicadores para robustecer el modelo. Además, la variable Transparencia se construye con las solicitudes de información, las cuales tienen una desviación estándar amplia, lo que puede generar asimetrias.

Es importante reconocer lo establecido por Sánchez de Diego-Fernández de la Riva y Sierra-Rodriguez (2020) quienes establecen la existencia de asimetrías en el grado de implantación de la transparencia, derivado principalmente por la voluntad política, el nivel de instrucción en la materia (quienes están al frente de la Unidades de Transparencia o de los órganos garantes), la disposición de recursos tecnológicos y de gestión, entre otros factores, lo que puede contribuir a los problemas planteados.

Se plantea para futuras investigaciones, la construcción de una gama más amplia de indicadores que permitan robustecer el modelo y, con ello, mejorar el coeficiente de determinación. Los indicadores sugeridos, entre otros son: las habilidades digitales, 
los niveles educativos más elevados, indicadores socioeconómicos, políticos o fiscales, como se han abordado en Mora-Pérez (2020). Además, establecer una noción más amplia de las relaciones entre la transparencia, el buen gobierno, la gobernanza, los datos abiertos y el propio gobierno abierto.

\section{Bibliografía}

Alt, J., y Lassen, D. (2006). Transparency, political polarization, and political budget cycles in OECD countries. Am J Polit Sci, 530-550.

Alt, J., Lassen, D., y Rose, S. (2006). The causes of fiscal transparency: evidence from the US States. International Monetary Fund.

Andreula, N., y Chong, A. (2015). Do good institutions improve fiscal transparency? . Econ Gov.

Bastida, F., y Benito, B. (2007). Central government budget practices and transparency: an international comparison. . Public Adm, 667-716.

Bernoth, K., y Wolff, G. B. (2008). Fool the Markets? Creative Accounting, Fiscal Transparency and Sovereign Risk Premia. Scottish Journal of Political Economy, 465-487.

Cabrero, E., y Nava, G. (2000). Gerencia Pública Municipal, conceptos básicos y estudios de caso. México: Porrúa.

Canales, J. M., y Romero-Tarín, A. (2017). Algunas consideraciones sobre la transparencia pública y el buen gobierno. Revista española de la transparencia, 115-127.

Cejudo, G., y Torres, N. (2020, 07 01). Retrieved from https://www.animalpolitico.com/intuiciones-y-evidencia/el-problema-de-lasinexistencias-en-el-acceso-a-la-informacion/

CIDE. (2014). Medición de Transparencia y Acceso a la Información en México . CIDE.

De Araujo, F., y Tejedo, F. (2016). Local government transparency index: determinants of municipalities rankings. International Journal of Public Sector Management.

De Renzio, P., y Wehner, J. (2015). The Impacts of Fiscal Openness: A Review of the Evidence. International Budget Parntneship.

Delgado, F. (2015). De qué hablamos cuando hablamos de transparencia. Revista española de la transparencia, 23-30.

Diputados, C. d. (2020, 07 01). Cámara de Diputados. Retrieved from http://www.diputados.gob.mx/LeyesBiblio/pdf/1_080520.pdf 
Diputados, C. d. (2020, 07 01). Cámara de Diputados. Retrieved from http://www.diputados.gob.mx/LeyesBiblio/pdf/LGTAIP.pdf

Drabek, Z., y Payne, W. (2001). The impact of transparency on foreign direct investment. WTO Staff Working Paper.

Estrada, Z. C., y Vargas-Hernández, J. (2018). Percepción Ciudadana del Open Data y la Innovación en México. RITI Journa, 86-95.

Federación, A. S. (2020, 07 01). Sistema Público de Consulta de Auditorías. Retrieved from http://www.asfdatos.gob.mx/

Fierro, A. E., y Gil-García, R. (2011). Más allá del acceso a la información. El uso de tecnologías de la información para fomentar la transparencia, la participación y la colaboración en el sector público. Documentos de trabajo, CIDE.

Fox, J., Haight, L., y Palmer-Rubin, B. (2011). Proporcionar transparencia: ¿Hasta qué punto responde el gobierno mexicano a las solicitudes de información pública? Gestión y Política Pública.

Gelos, G., y Wei, S.-J. (2002). Transparency and International Investor Behavior. IMF Working Papers.

Genesys. (2020, 04 09). Qué es un agente virtual. Retrieved from genesys.com/es/definitions/what-is-a-virtualagent.

Guillamon, M., Bastida, F., y Benito, B. (2011). The Determinants of Local Government's Financial Transparency. Local Government Studies, 391-406.

Hameed, F. (2005). Fiscal transparency and economic outcomes. International Monetary Fund.

Heald, D. (2013). Strengthening fiscal transparency. In R. Allen, R. Hemming, y B. Potter, The International Handbook of Public Financial Management. New York: Palgrave Macmillan.

INEGI. (2017). Estadísticas sobre transparencia y acceso a la información en México. En Números. Documentos de análisis y estadísticas.

INEGI. (2020, 07 01). Encuesta Nacional de Acceso a la Información Pública y Protección de Datos Personales (ENAID). Retrieved from https://www.inegi.org.mx/programas/enaid/2019/

INEGI. (2020, 07 01). Encuesta Nacional de Calidad e Impacto Gubernamental (ENCIG) . Retrieved from https://www.inegi.org.mx/programas/encig/2019/

INEGI. (2020, 07 07). Finanzas públicas estatales y municipales. Retrieved from https://www.inegi.org.mx/programas/finanzas/ 
Mora-Pérez, C. (2020). Determinantes de la Transparencia Fiscal en las Entidades Federativas de México. Guadalajara: Universidad de Guadalajara.

Nacional, S. E. (2020, 07 01). Instituto Nacional para la Evaluación de la Educación. Retrieved from https://www.inee.edu.mx/evaluaciones/bases-de-datos/

OCDE. (2005). Public Sector Modernization: Open Government. OCDE.

Philips, L., y Stewart, M. (2008). Defining Fiscal Transparency: Transitional Norms, Domestic Laws and the Politics of Budget Accountability. 4 CPLE Research Paper.

Sanchez de Diego-Fernandez de la Riva, M., y Sierra-Rodriguez, J. (2020). Transparencia y Participación para un Gobierno Abierto. Madrid, España: Wolters Kluwer.

Shin, Y., y Glennerster, R. (2003). Is Transparency Good for You, and Can the IMF Help? IMF Working Papers.

Sierra-Rodríguez, J. (2018). A modo de introducción: apuntes básicos sobre la ley de transparencia, acceso a la información pública y buen gobierno. In M. Sánchez de Diego Fernández de la Riva, Apuntes sobre la transparencia (pp. 13-32). Madrid: Universidad Complutense.

Sierra-Rodríguez, J. (2020). Estado de alarma, transparencia gubernamental y rendición de cuentas durante la crisis del COVID-19. In J. M. Reniu-Vilamala, y J. V. Meseguer-Sánchez, ¿Política confinada? Nuevas tecnologías y toma de decisiones en un contexto de pandemia (pp. 255-276). Madrid: Thomson Reuters.

SNT. (2020, 05 09). Sistema Nacional de Transparencia. Retrieved from http://www.snt.org.mx/index.php/informacioninteres/estadisticas

Tavares, A., y Ferreira, N. (2014). The Determinants of Local Government Transparency: A Preliminary Analysis. In ICEGOV, 8th International Conference on Theory and Practice of Electronic Governance. Portugal: ICEGOV.

Teig, M. (2009). Fiscal Transparency and Economic Growth. Germany: Bamberg University.

Villoria-Mendieta, M. (2014). Desarrollo, buen gobierno y transparencia: revisión conceptual. Revista Tiempo de Paz, 4-10.

Zuccolotto, R., y Teixeira, M. (2014). The Causes of Fiscal Transparency: Evidence in the Brazilian States. Cont. Fin. - USP, 242-254. 